\title{
A DERIVATION OF THE HARDY-RAMANUJAN FORMULA FROM AN ARITHMETIC FORMULA
}

\author{
MICHAEL DEWAR AND M. RAM MURTY \\ (Communicated by Ken Ono)
}

\begin{abstract}
We re-prove the Hardy-Ramanujan asymptotic formula for the partition function without using the circle method. We derive our result from recent work of Bruinier and Ono on harmonic weak Maass forms.
\end{abstract}

\section{INTRODUCTION}

The partition function $p(n)$ counts the number of ways to write $n$ as a nonincreasing sequence of positive integers. Hardy and Ramanujan [6] created the circle method to prove the asymptotic formula

$$
p(n) \sim \frac{1}{4 n \sqrt{3}} \cdot e^{\pi \sqrt{\frac{2 n}{3}}} .
$$

Rademacher [7], and later Selberg [8] independently, pushed the circle method further to obtain the exact formula

$$
p(n)=\frac{1}{\pi \sqrt{2}} \sum_{m=1}^{\infty} \sqrt{m} A_{m}(n) \frac{d}{d n}\left[\frac{\sinh \left(\frac{\pi}{m} \sqrt{\frac{2}{3}\left(1-\frac{1}{24}\right)}\right)}{\sqrt{n-\frac{1}{24}}}\right],
$$

or equivalently,

$$
\begin{aligned}
(24 n-1) p(n)=\sum_{m=1}^{\infty} 2 \sqrt{\frac{3}{m}} A_{m}(n) & \left(1-\frac{6 m}{\pi \sqrt{24 n-1}}\right) e^{\frac{\pi \sqrt{24 n-1}}{6 m}} \\
& +2 \sqrt{\frac{3}{m}} A_{m}(n)\left(1+\frac{6 m}{\pi \sqrt{24 n-1}}\right) e^{-\frac{\pi \sqrt{24 n-1}}{6 m}}
\end{aligned}
$$

where $A_{m}(n)$ is a Kloosterman sum (see 7]).

We use the algebraic formula for $p(n)$ of Bruinier and Ono [1] (Theorem 4 below) to prove an asymptotic formula which captures the main terms of (1.2) without using the circle method. In particular, we prove an asymptotic formula of the form

$$
(24 n-1) p(n)=\sum_{m=1}^{N} c_{m}\left(1-\frac{6 m}{\pi \sqrt{24 n-1}}\right) e^{\frac{\pi \sqrt{24 n-1}}{6 m}}+O\left(h(1-24 n) e^{\frac{\pi \sqrt{24 n-1}}{6(N+1)}}\right),
$$

Received by the editors September 22, 2011.

2010 Mathematics Subject Classification. Primary 11E16, 11F03, 11 P82.

The first author was supported by a postdoctoral fellowship from the Natural Sciences and Engineering Council of Canada (NSERC).

The second author was supported by a Discovery Grant from NSERC. 
where the $c_{m}$ depend on the congruence classes of $n(\bmod m)$ and $h(1-24 n)$ is the usual class number of discriminant $1-24 n$. We define a sequence of functions

$$
c_{m}(n): \mathbb{Z} / m \mathbb{Z} \rightarrow \mathbb{R}
$$

and implicitly provide a straightforward algorithm to compute $c_{m}(n)$. In particular, $c_{m}(n)$ is a finite sum of $12 m^{\text {th }}$ roots of unity. For example,

$$
\begin{gathered}
c_{1}(n)=2 \sqrt{3}, \quad c_{2}(n)=2(-1)^{n}\left(\cos \left(\frac{\pi}{12}\right)+\cos \left(\frac{5 \pi}{12}\right)\right), \\
c_{3}(n)=\left\{\begin{array}{lll}
4 \cos \left(\frac{\pi}{18}\right) & \text { if } n \equiv 0 \quad(\bmod 3) \\
4 \cos \left(\frac{11 \pi}{18}\right) & \text { if } n \equiv 1 \quad(\bmod 3), \\
4 \cos \left(\frac{23 \pi}{18}\right) & \text { if } n \equiv 2 \quad(\bmod 3)
\end{array}\right. \\
c_{4}(n)=\left\{\begin{array}{lll}
2 \cos \left(\frac{\pi}{24}\right)+2 \cos \left(\frac{7 \pi}{24}\right) & \text { if } n \equiv 0 \quad(\bmod 4) \\
2 \cos \left(\frac{5 \pi}{24}\right)+2 \cos \left(\frac{13 \pi}{24}\right) & \text { if } n \equiv 1 \quad(\bmod 4) \\
-2 \cos \left(\frac{\pi}{24}\right)-2 \cos \left(\frac{7 \pi}{24}\right) & \text { if } n \equiv 2 \quad(\bmod 4) \\
-2 \cos \left(\frac{5 \pi}{24}\right)-2 \cos \left(\frac{13 \pi}{24}\right) & \text { if } n \equiv 3 \quad(\bmod 4)
\end{array}\right.
\end{gathered}
$$

Theorem 1. Let $N \geq 1$ and $1 \leq r \leq \operatorname{lcm}[1,2, \ldots, N]$ and take $c_{m}(r)$ as defined in Section 4. If $n \equiv r(\bmod \operatorname{lcm}[1,2, \ldots, N])$ and $n \geq 6 N^{2}$, then

$$
(24 n-1) p(n)=\sum_{m=1}^{N} c_{m}(r)\left(1-\frac{6 m}{\pi \sqrt{24 n-1}}\right) e^{\frac{\pi \sqrt{24 n-1}}{6 m}}+O\left(h(1-24 n) e^{\frac{\pi \sqrt{24 n-1}}{6(N+1)}}\right),
$$

where the implied constant is absolute.

Although it is not immediately obvious from the definitions, we must have $c_{m}(n)=2 \sqrt{\frac{3}{m}} A_{m}(n)$.

Since $h(1-24 n)$ grows subexponentially, (1.1) is a corollary to Theorem 1

\section{Corollary 2.}

$$
p(n) \sim \frac{1}{4 n \sqrt{3}} \cdot e^{\pi \sqrt{\frac{2 n}{3}}} .
$$

Proof. Take $N=1$ in the theorem. Then since $1 / \sqrt{24 n-1}=o(1)$, we have

$$
p(n)=\frac{2 \sqrt{3}}{24 n-1} \cdot e^{\frac{\pi \sqrt{24 n-1}}{6}}+o\left(e^{\frac{\pi \sqrt{24 n-1}}{6}}\right) .
$$

The conclusion follows.

In Section 2 we establish notation related to binary quadratic forms. These preliminaries are necessary for Section 3 in which we recall Bruinier and Ono's work [1] on harmonic weak Maass forms. Finally, in Section 4 we prove Theorem 1]

\section{BINARY QUADRATIC FORMS}

We set notation and recall the fundamentals of positive definite quadratic forms. There are many good introductions to this classical material; see 4 for an example.

An integral binary quadratic form $Q(x, y)=a x^{2}+b x y+c y^{2}$ with discriminant $b^{2}-4 a c$ is called primitive if $\operatorname{gcd}(a, b, c)=1$. We will often write $Q=[a, b, c]$. Throughout this article, we restrict our attention to positive definite forms with 
discriminant $1-24 n$ for positive integers $n$. It is elementary to see that for such forms $0 \equiv a c \not \equiv b(\bmod 2)$ and

$$
\begin{aligned}
& 3 \mid b \Longleftrightarrow a c \equiv 2 \quad(\bmod 6), \\
& 3 \nmid b \Longleftrightarrow a c \equiv 0 \quad(\bmod 6) .
\end{aligned}
$$

Let $\mathbb{H}$ denote the upper half of the complex plane. The principal root of $Q=$ $[a, b, c]$ is the unique point $\alpha=-\frac{b}{2 a}+\frac{\sqrt{24 n-1}}{2 a} i \in \mathbb{H}$ such that $Q(\alpha, 1)=0$. Matrices $\left(\begin{array}{ll}r & s \\ t & u\end{array}\right) \in \mathrm{SL}_{2}(\mathbb{Z})$ act on $z \in \mathbb{H}$ via the left action

$$
\left(\begin{array}{cc}
r & s \\
t & u
\end{array}\right) \circ z=\frac{r z+s}{t z+u}
$$

and on forms via the right action

$$
Q \circ\left(\begin{array}{cc}
r & s \\
t & u
\end{array}\right)(x, y)=Q(r x+s y, t x+u y),
$$

which preserves the discriminant. If $\alpha$ is the principal root of $Q$ and $\gamma \in \mathrm{SL}_{2}(\mathbb{Z})$, then $\gamma \circ \alpha$ is the principal root of $Q \circ \gamma^{-1}$.

We say that $Q=[a, b, c]$ is $\mathrm{SL}_{2}(\mathbb{Z})$-reduced if $|b| \leq a \leq c$. Every positive definite form is $\mathrm{SL}_{2}(\mathbb{Z})$-equivalent to a reduced form. With the exception of $[a, b, a] \sim$ $[a,-b, a]$ and $[a, a, c] \sim[a,-a, c]$, no distinct reduced forms are $\mathrm{SL}_{2}(\mathbb{Z})$-equivalent. The (finite) number of $\mathrm{SL}_{2}(\mathbb{Z})$-equivalence classes of primitive binary quadratic forms of discriminant $1-24 n$ is called the class number $h(1-24 n)$. A form is reduced if and only if its principal root lies in the closure of the usual fundamental domain for $\mathrm{SL}_{2}(\mathbb{Z})$,

$\mathcal{F}=\left\{z \in \mathbb{H}:-\frac{1}{2} \leq \operatorname{Re} z<\frac{1}{2}\right.$ and $\left.|z|>1\right\} \cup\left\{z \in \mathbb{H}:|z|=1\right.$ and $\left.-\frac{1}{2} \leq \operatorname{Re} z \leq 0\right\}$.

Let $\mathcal{Q}_{n}^{1}$ denote the set of primitive forms of discriminant $1-24 n$ whose principal roots lie in $\mathcal{F}$. Observe that $\mathcal{Q}_{n}^{1}$ does not double count any $\mathrm{SL}_{2}(\mathbb{Z})$-equivalence class.

The congruence group $\Gamma_{0}(6)=\left\{\left(\begin{array}{cc}r & s \\ t & u\end{array}\right) \in \mathrm{SL}_{2}(\mathbb{Z}): 6 \mid t\right\}$ acts on the set of primitive forms $[a, b, c]$ of discriminant $1-24 n$ such that $6 \mid a$ and $b \equiv 1(\bmod 12)$. Let $\mathcal{Q}_{n}^{6}$ denote the set of $\Gamma_{0}(6)$-equivalence classes of such forms. The group $\Gamma_{0}(6)$ has index $\left[\mathrm{SL}_{2}(\mathbb{Z}): \Gamma_{0}(6)\right]=12$, and we choose right coset representatives:

$$
\begin{aligned}
\gamma_{\infty} & =\left(\begin{array}{ll}
1 & 0 \\
0 & 1
\end{array}\right) \\
\gamma_{\frac{1}{3}, r} & =\left(\begin{array}{ll}
1 & 0 \\
3 & 1
\end{array}\right)\left(\begin{array}{ll}
1 & r \\
0 & 1
\end{array}\right) \text { for } r=0,1 \\
\gamma_{\frac{1}{2}, s} & =\left(\begin{array}{ll}
1 & 1 \\
2 & 3
\end{array}\right)\left(\begin{array}{ll}
1 & s \\
0 & 1
\end{array}\right) \text { for } s=0,1,2, \\
\gamma_{0, t} & =\left(\begin{array}{cc}
0 & -1 \\
1 & 0
\end{array}\right)\left(\begin{array}{ll}
1 & t \\
0 & 1
\end{array}\right) \text { for } t=0,1,2,3,4,5 .
\end{aligned}
$$

The proposition on page 505 of [5] says that there is a bijection between $\mathcal{Q}_{n}^{1}$ and $\mathcal{Q}_{n}^{6}$. In particular, for any $Q \in \mathcal{Q}_{n}^{1}$, there exists a unique choice from (2.1) of right coset leader $\gamma_{Q}$ such that $\left[Q \circ \gamma_{Q}^{-1}\right] \in \mathcal{Q}_{n}^{6}$. It will be expedient to know $\gamma_{Q}$ explicitly. The proof of the following lemma obviates the appeal to [5]. 
TABLE 1. The matrix $\gamma_{Q}$ for $Q=[a, b, c] \in \mathcal{Q}_{n}^{1}$. Triples $(\bar{a}, \bar{b}, \bar{c})$ which can never occur are omitted or left blank.

\begin{tabular}{|c|c|c|c|c|c|c|c|}
\hline & \multicolumn{6}{|c|}{$b(\bmod 12)$} \\
\hline & & 1 & 3 & 5 & 7 & 9 & 11 \\
\hline \multirow{6}{*}{$a \equiv 0(\bmod 6)$} & 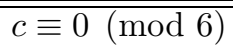 & $\gamma_{\infty}$ & & $\gamma_{\frac{1}{2}, 0}$ & $\gamma_{\frac{1}{3}, 1}$ & & $\gamma_{0,0}$ \\
\hline & $c \equiv 1(\bmod 6)$ & $\gamma_{\infty}$ & & $\gamma_{\frac{1}{2}, 2}$ & $\gamma_{\frac{1}{3}, 0}$ & & $\gamma_{0,5}$ \\
\hline & $c \equiv 2(\bmod 6)$ & $\gamma_{\infty}$ & & $\gamma_{\frac{1}{2}, 1}$ & $\gamma_{\frac{1}{3}, 1}$ & & $\gamma_{0,4}$ \\
\hline & $c \equiv 3(\bmod 6)$ & $\gamma_{\infty}$ & & $\gamma_{\frac{1}{2}, 0}$ & $\gamma_{\frac{1}{3}, 0}$ & & $\gamma_{0,3}$ \\
\hline & $c \equiv 4(\bmod 6)$ & $\gamma_{\infty}$ & & $\gamma_{\frac{1}{2}, 2}$ & $\gamma_{\frac{1}{3}, 1}$ & & $\gamma_{0,2}$ \\
\hline & $c \equiv 5(\bmod 6)$ & $\gamma_{\infty}$ & & $\gamma_{\frac{1}{2}, 1}$ & $\gamma_{\frac{1}{3}, 0}$ & & $\gamma_{0,1}$ \\
\hline \multirow{2}{*}{$a \equiv 1(\bmod 6)$} & $c \equiv 0(\bmod 6)$ & $\gamma_{0,1}$ & & $\gamma_{0,3}$ & $\gamma_{0,4}$ & & $\gamma_{0,0}$ \\
\hline & $c \equiv 2(\bmod 6)$ & & $\gamma_{0,2}$ & & & $\gamma_{0,5}$ & \\
\hline \multirow{4}{*}{$a \equiv 2(\bmod 6)$} & $c \equiv 0(\bmod 6)$ & $\gamma_{\frac{1}{2}, 2}$ & & $\gamma_{\frac{1}{2}, 0}$ & $\gamma_{0,2}$ & & $\gamma_{0,0}$ \\
\hline & $c \equiv 1(\bmod 6)$ & & $\gamma_{0,1}$ & & & $\gamma_{\frac{1}{2}, 1}$ & \\
\hline & $c \equiv 3(\bmod 6)$ & $\gamma_{\frac{1}{2}, 2}$ & & $\gamma_{\frac{1}{2}, 0}$ & $\gamma_{0,5}$ & & $\gamma_{0,3}$ \\
\hline & $c \equiv 4(\bmod 6)$ & & $\gamma_{0,4}$ & & & $\gamma_{\frac{1}{2}, 1}$ & \\
\hline \multirow{3}{*}{$a \equiv 3(\bmod 6)$} & $c \equiv 0(\bmod 6)$ & $\gamma_{\frac{1}{3}, 0}$ & & $\gamma_{0,3}$ & $\gamma_{\frac{1}{3}, 1}$ & & $\gamma_{0,0}$ \\
\hline & $c \equiv 2(\bmod 6)$ & $\gamma_{\frac{1}{3}, 0}$ & & $\gamma_{0,1}$ & $\gamma_{\frac{1}{3}, 1}$ & & $\gamma_{0,4}$ \\
\hline & $c \equiv 4(\bmod 6)$ & $\gamma_{\frac{1}{3}, 0}$ & & $\gamma_{0,5}$ & $\gamma_{\frac{1}{3}, 1}$ & & $\gamma_{0,2}$ \\
\hline \multirow{4}{*}{$a \equiv 4(\bmod 6)$} & $c \equiv 0(\bmod 6)$ & $\gamma_{\frac{1}{2}, 1}$ & & $\gamma_{\frac{1}{2}, 0}$ & $\gamma_{0,4}$ & & $\gamma_{0,0}$ \\
\hline & $c \equiv 2(\bmod 6)$ & & $\gamma_{0,2}$ & & & $\gamma_{\frac{1}{2}, 2}$ & \\
\hline & $c \equiv 3(\bmod 6)$ & $\gamma_{\frac{1}{2}, 1}$ & & $\gamma_{\frac{1}{2}, 0}$ & $\gamma_{0,1}$ & & $\gamma_{0,3}$ \\
\hline & $c \equiv 5(\bmod 6)$ & & $\gamma_{0,5}$ & & & $\gamma_{\frac{1}{2}, 2}$ & \\
\hline \multirow{2}{*}{$a \equiv 5(\bmod 6)$} & $c \equiv 0(\bmod 6)$ & $\gamma_{0,5}$ & & $\gamma_{0,3}$ & $\gamma_{0,2}$ & & $\gamma_{0,0}$ \\
\hline & $c \equiv 4(\bmod 6)$ & & $\gamma_{0,4}$ & & & $\gamma_{0,1}$ & \\
\hline
\end{tabular}

Lemma 3. If $Q=[a, b, c] \in \mathcal{Q}_{n}^{1}$, then the matrix $\gamma_{Q}$ from (2.1) such that $\left[Q \circ \gamma_{Q}^{-1}\right] \in$ $\mathcal{Q}_{n}^{6}$ is as given in Table 1 .

Proof. Consider $\gamma_{\frac{1}{2}, 0}=\left(\begin{array}{ll}1 & 1 \\ 2 & 3\end{array}\right)$. We compute that $[a, b, c] \circ \gamma_{\frac{1}{2}, 0}^{-1}=[A, B, C]$, where

$$
\begin{aligned}
& A=9 a-6 b+4 c, \\
& B=-6 a+5 b-4 c .
\end{aligned}
$$

Clearly $2|a, 3| c$ and $b \equiv 5(\bmod 12)$ if and only if $A \equiv 0(\bmod 6)$ and $B \equiv 1$ $(\bmod 12)$. The latter conditions are precisely the requirements for $[A, B, C]=$ $[a, b, c] \circ \gamma_{\frac{1}{2}, 0}^{-1} \in \mathcal{Q}_{n}^{6}$. This gives six entries in Table 1, The other 11 matrices from (2.1) are analogous.

\section{HARMONIC WEAK MAASS FORMS}

We recall notation from [1. We adopt the convention that $z=x+i y \in \mathbb{H}$ with $x, y \in \mathbb{R}$. The Maass raising operator (see Section 2 of [2] $R_{k}$ acts on functions $f: \mathbb{H} \rightarrow \mathbb{C}$ and is defined by

$$
R_{k}=2 i \frac{\partial}{\partial z}+\frac{k}{y}=i\left(\frac{\partial}{\partial x}-i \frac{\partial}{\partial y}\right)+\frac{k}{y} .
$$


Given $\gamma=\left(\begin{array}{ll}r & s \\ t & u\end{array}\right) \in \mathrm{SL}_{2}(\mathbb{Z})$, we define the Petersson slash operator which also acts on functions $f: \mathbb{H} \rightarrow \mathbb{C}$ by

$$
\left.f\right|_{k} \gamma=(t z+u)^{-k} f\left(\frac{r z+s}{t z+u}\right) .
$$

The slash operator intertwines with the raising operator

$$
R_{k}\left(\left.f\right|_{k} \gamma\right)=\left.\left(R_{k} f\right)\right|_{k+2} \gamma \text {. }
$$

Recall Dedekind's eta function $\eta(z):=q^{1 / 24} \prod_{n=1}^{\infty}\left(1-q^{n}\right)$, where $q=e^{2 \pi i z}$ and the Eisenstein series $E_{2}(z):=1-24 \sum_{n=1}^{\infty} \sigma(n) q^{n}$, where $\sigma(n)=\sum_{d \mid n} d$. Define the $\Gamma_{0}(6)$ weight -2 weakly holomorphic modular form

$$
F(z):=\frac{1}{2} \cdot \frac{E_{2}(z)-2 E_{2}(2 z)-3 E_{2}(3 z)+6 E_{2}(6 z)}{\eta^{2}(z) \eta^{2}(2 z) \eta^{2}(3 z) \eta^{2}(6 z)}=q^{-1}-10-29 q-\cdots .
$$

Set $\zeta_{6}:=e^{2 \pi i / 6}$. Fourier expansions of $F$ at the cusps $\frac{1}{3}, \frac{1}{2}$ and 0 are given by

$$
\begin{aligned}
\left.F\right|_{-2} \gamma_{\frac{1}{3}, r} & =2(-1)^{r} q^{-1 / 2}+20-(-1)^{r} 34 q^{1 / 2}+\cdots, \\
\left.F\right|_{-2} \gamma_{\frac{1}{2}, s} & =3 \zeta_{6}^{3-2 s} q^{-1 / 3}+30-87 \zeta_{6}^{3+2 s} q^{1 / 3}+\cdots, \\
\left.F\right|_{-2} \gamma_{0, t} & =6 \zeta_{6}^{-t} q^{-1 / 6}-60-174 \zeta_{6}^{t} q^{1 / 6}-\cdots .
\end{aligned}
$$

For any $Q \in \mathcal{Q}_{n}^{1}$, define $h_{Q} \in\{1,2,3,6\}$ to be the width of the cusp $\gamma_{Q} \circ \infty$ on the modular curve $X_{0}(6)$ and define $\zeta_{Q}$ to be the sixth root of unity such that

$$
\left.F\right|_{-2} \gamma_{Q}=h_{Q} \zeta_{Q} q^{-1 / h_{Q}}+O(1) .
$$

Bruinier and Ono define the $\Gamma_{0}(6)$ weight zero weak Maass form

$$
P(z):=\frac{1}{4 \pi} R_{-2} F(z)=\left(1-\frac{1}{2 \pi y}\right) q^{-1}+\frac{5}{\pi y}+\left(29+\frac{29}{2 \pi y}\right) q+\cdots .
$$

Part of Theorem 1.1 of [1] says:

Theorem 4 (Bruinier-Ono). For all positive integers n,

$$
(24 n-1) p(n)=\sum_{[Q] \in \mathcal{Q}_{n}^{6}} P\left(\alpha_{Q}\right),
$$

where $\alpha_{Q}$ denotes the principal root of $Q$.

Ono has informed us that an upcoming article 3] will use the theory of elliptic curves with complex multiplication to compute the polynomials whose roots are the singular moduli for $P(z)$ modulo primes $p$. By the Chinese Remainder Theorem, they then obtain a fast algorithm for deriving these polynomials exactly.

\section{Proof of Theorem 1}

The key to the proof of Theorem 1 is that if $P\left(\alpha_{Q}\right)$ is evaluated using a Fourier expansion centered at the cusp closest - on $X_{0}(6)$ - to $\alpha_{Q}$, then the first term of the Fourier expansion dominates the tail.

Lemma 5. Let $\gamma$ be one of the 12 matrices from (2.1) and suppose that

$$
\left(\left.F\right|_{-2} \gamma\right)(z)=h \zeta q^{-1 / h}+a(0)+a(1) q^{1 / h}+\cdots,
$$


where $h$ is the width of the cusp $\gamma \circ \infty$ on the modular curve $X_{0}(6)$ and $\zeta \in \mathbb{C}$. Then for $z \in \mathcal{F}$,

$$
\left(\left.P\right|_{0} \gamma\right)(z)=\zeta\left(1-\frac{h}{2 \pi y}\right) e^{-2 \pi i z / h}+O(1),
$$

where the implied constant is absolute.

Proof. The intertwining property (3.1) of $R_{-2}$ implies that

$$
\begin{aligned}
\left.P\right|_{0} \gamma & =\left.\left(\frac{1}{4 \pi} R_{-2} F\right)\right|_{0} \gamma \\
& =\frac{1}{4 \pi} R_{-2}\left(\left.F\right|_{-2} \gamma\right) \\
& =\frac{1}{4 \pi} R_{-2}\left(h \zeta q^{-1 / h}+\left(\left.F\right|_{-2} \gamma-h \zeta q^{-1 / h}\right)\right) \\
& =\zeta\left(1-\frac{h}{2 \pi y}\right) e^{-2 \pi i z / h}+\frac{1}{4 \pi} R_{-2}\left(\left.F\right|_{-2} \gamma-h \zeta q^{-1 / h}\right) .
\end{aligned}
$$

Thus, it suffices to show that the second term is bounded by a constant depending only on $\gamma$, since there are only 12 possible choices for $\gamma$.

Suppose $z \in \mathcal{F}$. Then clearly

$$
z \in \mathcal{U}:=\left\{z \in \mathbb{H}:-\frac{h}{2} \leq \operatorname{Re} z<\frac{h}{2}, \operatorname{Im} z \geq \frac{\sqrt{3}}{2}\right\} .
$$

Recall that the Fourier expansion of the meromorphic modular form $F(z)$ at a cusp of width $h$ is in fact a Laurent expansion of a meromorphic function on a punctured disc in the local variable $q_{h}:=q^{1 / h}=e^{2 \pi i z / h}$. Hence, $f\left(q_{h}\right):=\left.F\right|_{-2} \gamma-h \zeta q_{h}^{-1}$ is bounded on the closure of the punctured disc. Since $F(z)$ is holomorphic for all $z \in \mathbb{H}$, the $q_{h}$-series expansion of $f$ converges for all $\left|q_{h}\right|<1$. In particular, $f\left(q_{h}\right)$ is bounded on $\left\{q_{h}:\left|q_{h}\right| \leq e^{-\pi \sqrt{3} / h}\right\}$.

Now $-\frac{1}{2 \pi i} \frac{d}{d z}=\frac{1}{h} q_{h} \frac{d}{d q_{h}}$, and hence

$$
-\frac{1}{2 \pi i} \frac{d}{d z}\left(\left.F\right|_{-2} \gamma-h \zeta q^{-1 / h}\right)=\frac{1}{h} q_{h} \frac{d}{d q_{h}} f\left(q_{h}\right)
$$

is holomorphic, and hence bounded, for $\left|q_{h}\right| \leq e^{-\pi \sqrt{3} / h}$, and hence for $z \in \mathcal{F} \subset \mathcal{U}$. Additionally, $1 /(2 \pi y)$ is bounded on $\mathcal{U}$. Hence, for $z \in \mathcal{U}$, we have

$$
\frac{1}{4 \pi} R_{-2}\left(\left.F\right|_{-2} \gamma-h \zeta q^{-1 / h}\right)=O(1)
$$

We let $\alpha_{Q}$ denote the principal root of $Q$. Now by the explicit bijection $\mathcal{Q}_{n}^{1} \leftrightarrow$ $\mathcal{Q}_{n}^{6}$ and Theorem 4, we have

$$
(24 n-1) p(n)=\sum_{[Q] \in \mathcal{Q}_{n}^{6}} P\left(\alpha_{Q}\right)=\sum_{Q \in \mathcal{Q}_{n}^{1}} P\left(\gamma_{Q} \circ \alpha_{Q}\right)=\sum_{Q \in \mathcal{Q}_{n}^{1}}\left(\left.P\right|_{0} \gamma_{Q}\right)\left(\alpha_{Q}\right) .
$$


The widths of the cusps $\gamma_{\infty} \circ \infty, \gamma_{\frac{1}{3}, r} \circ \infty, \gamma_{\frac{1}{2}, s} \circ \infty$, and $\gamma_{0, t} \circ \infty$, are, respectively, $1,2,3$, and 6. Observe from Table 1 that for any $Q=[a, b, c] \in \mathcal{Q}_{n}^{1}$, the product

$$
a \cdot h_{Q} \equiv 0 \quad(\bmod 6)
$$

We group the summands in (4.1) accordingly and apply Lemma 5 using $\alpha_{Q}=$ $-\frac{b}{2 a}+\frac{\sqrt{24 n-1}}{2 a} i$ :

$$
\begin{aligned}
(24 n-1) p(n)= & \sum_{m=1}^{\infty} \sum_{\substack{[a, b, c] \in \mathcal{Q}_{n}^{1} \\
a \cdot h_{[a, b, c]}=6 m}}\left(\left.P\right|_{0} \gamma_{Q}\right)\left(\alpha_{Q}\right) \\
= & \sum_{m=1}^{\infty}\left(\sum_{\substack{[a, b, c] \in \mathcal{Q}_{n}^{1} \\
a \cdot h_{[a, b, c]}=6 m}} \zeta_{[a, b, c]} e^{\pi i b / 6 m}\right)\left(1-\frac{6 m}{\pi \sqrt{24 n-1}}\right) e^{\frac{\pi \sqrt{24 n-1}}{6 m}} \\
& +O(h(1-24 n)) \\
= & \sum_{m=1}^{N}\left(\begin{array}{c}
\sum_{\substack{[a, b, c] \in \mathcal{Q}_{n}^{1} \\
a \cdot h_{[a, b, c]}=6 m}} \zeta_{[a, b, c]} e^{\pi i b / 6 m} \\
\\
+
\end{array}\right)\left(1-\frac{6 m}{\pi \sqrt{24 n-1}}\right) e^{\frac{\pi \sqrt{24 n-1}}{6 m}} \\
& \left.O(1-24 n) e^{\frac{\pi \sqrt{24 n-1}}{6(N+1)}}\right) .
\end{aligned}
$$

The truncation in the last step omits at most $h(1-24 n)$ summands, each of which is clearly bounded by $e^{\frac{\pi \sqrt{24 n-1}}{6(N+1)}} \geq 1$. Hence the implied constants are still absolute. It remains to consider the finite sums

$$
\widehat{c_{m}}(n):=\sum_{\substack{[a, b, c] \in \mathcal{Q}_{n}^{1} \\ a \cdot h_{[a, b, c]}=6 m}} \zeta_{[a, b, c]} e^{\pi i b / 6 m} .
$$

As an example, we first consider $\widehat{c_{1}}(n)$. It is easy to determine that the forms $[a, b, c] \in \mathcal{Q}_{n}^{1}$ with $a \leq 6$ are exactly:

$$
\begin{aligned}
& {[1,1,6 n]} \\
& {[2,1,3 n]} \\
& \text { if } n \equiv 0(\bmod 5)\left\{\begin{array}{c}
{\left[5,1, \frac{6 n}{5}\right]} \\
{\left[5,-1, \frac{6 n}{5}\right]}
\end{array}\right. \\
& {[2,-1,3 n]} \\
& {[3,1,2 n]} \\
& {[3,-1,2 n]} \\
& \text { if } n \text { even }\left\{\begin{array}{r}
{\left[4,1, \frac{3 n}{2}\right]} \\
{\left[4,-1, \frac{3 n}{2}\right]}
\end{array}\right. \\
& \text { if } n \equiv 3(\bmod 5)\left\{\begin{array}{c}
{\left[5,3, \frac{6 n+2}{5}\right]} \\
{\left[5,-3, \frac{6 n+2}{5}\right]}
\end{array}\right. \\
& \text { if } n \equiv 4(\bmod 5)\left\{\quad\left[5,5, \frac{6 n+6}{5}\right]\right. \\
& \text { if } n \text { odd }\left\{\begin{array}{c}
{\left[4,3, \frac{3 n+1}{2}\right]} \\
{\left[4,-3, \frac{3 n+1}{2}\right]}
\end{array}\right. \\
& {[6,1, n]} \\
& {[6,-1, n]} \\
& {[6,5, n+1]} \\
& {[6,-5, n+1] \text {. }}
\end{aligned}
$$


For each of these, Table 1 indicates the $\gamma_{Q}$ for which $Q \circ \gamma_{Q}^{-1}=[A, B, C] \in \mathcal{Q}_{n}^{6}$. For example,

$$
\left.\begin{array}{rl}
{[1,1,6 n] \circ \gamma_{0,1}^{-1}} & =[6 n, 1,1] \\
{[2,1,3 n] \circ \gamma_{\frac{1}{2},-1}^{-1}} & =[84+12 n,-71-12 n, 15+3 n] \\
{[3,1,2 n] \circ \gamma_{\frac{1}{3}, 0}^{-1}} & =[126+18 n,-71-12 n, 10+2 n] \\
{[6,1, n] \circ \gamma_{\infty}} & =[6,1, n]
\end{array}\right\} \in \mathcal{Q}_{n}^{6} .
$$

For each of these four forms, we see that $a h=6$. Moreover, it is easy to check that for all other forms, we have $a h \geq 12$. That is, for all $Q \in \mathcal{Q}_{n}^{6}$ other than the four listed in (4.2), we have

$$
P\left(\alpha_{Q}\right)=O\left(e^{\pi \frac{\sqrt{24 n-1}}{12}}\right) .
$$

Finally, we compute that if $n \geq 2$, we have

$$
\widehat{c_{1}}(n)=\zeta_{6}^{-1} \cdot e^{\pi i / 6}+\zeta_{6}^{3-2(-1)} \cdot e^{\pi i / 6}+(-1)^{0} \cdot e^{\pi i / 6}+1 \cdot e^{\pi i / 6}=4 \cos \left(\frac{\pi}{6}\right)=2 \sqrt{3} \text {. }
$$

More generally, we have the following lemma.

Lemma 6. If $n \geq 6 m^{2}$, then $\widehat{c_{m}}(n)$ only depends on $n(\bmod m)$.

Proof. For a given $m$, we consider forms with $a=m, 2 m, 3 m$, and $6 m$. From Table 1 we see that $h_{[a, b, c]}$ depends only on $a(\bmod 6)$ and $b(\bmod 12)$. Thus, for each of the four choices of $a$, we consider all $b$ with $|b| \leq a$ and such that $b(\bmod 12)$ would yield the proper width $h_{[a, b, *]}=6 \mathrm{~m} / a$. We stress that the set of pairs of $a$ and $b$ which we consider depends only on $m$.

For each pair $a$ and $b$, the only possible $c$ for which $b^{2}-4 a c=1-24 n$ is obviously

$$
c=\frac{6 n+\left(\frac{b^{2}-1}{4}\right)}{a} .
$$

Thus, $[a, b, c] \in \mathcal{Q}_{n}^{1}$ if and only if $6 n+\left(\frac{b^{2}-1}{4}\right) \equiv 0(\bmod a)$ and $6 n+\left(\frac{b^{2}-1}{4}\right) \geq a^{2}$. The latter condition is satisfied whenever $n \geq 6 m^{2}$. By (3.2) and Lemma 3, if $[a, b, c] \in \mathcal{Q}_{n}^{1}$, then $\zeta_{[a, b, c]}$ depends on $c(\bmod h)$. Thus, the contribution of the pair $a$ and $b$, if any, to $\widehat{c_{m}}(n)$ depends on $6 n$ modulo $a h=6 m$. That is, $\widehat{c_{m}}(n)$ depends only on $n(\bmod m)$.

In light of the previous lemma, $\widehat{c_{m}}(n)$ induces (in the obvious way) the function

$$
c_{m}(n): \mathbb{Z} / m \mathbb{Z} \rightarrow \mathbb{C}
$$

referred to in Section 1. To see that $c_{m}(n)$ in fact maps to $\mathbb{R}$, we take real parts of the asymptotic expansion for $\operatorname{Re}((24 n-1) p(n))=(24 n-1) p(n)$. This concludes the proof of Theorem 1 .

\section{REFERENCES}

1. J. H. Bruinier and K. Ono. Algebraic formulas for the coefficients of half-integral weight harmonic weak Maass forms. arXiv:1104.1182, Apr. 2011.

2. J. H. Bruinier, K. Ono, and R. C. Rhoades. Differential operators for harmonic weak Maass forms and the vanishing of Hecke eigenvalues. Math. Ann., 342(3):673-693, 2008. MR.2430995 (2009f:11046) 
3. J. H. Bruinier, K. Ono, and A. Sutherland. Class polynomials for nonholomorphic modular functions, in preparation.

4. D. Buell. Binary Quadratic Forms. Springer-Verlag, 1989. MR1012948 (92b:11021)

5. B. Gross, W. Kohnen, and D. Zagier. Heegner points and derivatives of $L$-series. II. Mathematische Annalen, 278:497-562, 1987. MR909238 (89i:11069)

6. G. H. Hardy and S. Ramanujan. Asymptotic formulae in combinatory analysis. Proc. London Math. Soc. (2), 17:75-115, 1918.

7. H. Rademacher. On the partition function $p(n)$. Proc. London Math. Soc. (2), 43:241-254, 1937.

8. A. Selberg. Reflections around the Ramanujan centenary. In Collected Papers, Volume 1, pages 695-706. Springer-Verlag, 1989. MR 1117906 (92h:01083)

Department of Mathematics, Queen's University, Kingston, Ontario, K7L 3N6, CANADA

E-mail address: mdewar@mast.queensu.ca

Department of Mathematics, Queen's University, Kingston, Ontario, K7L 3N6, CANADA

E-mail address: murty@mast.queensu.ca 